\title{
Journal of

\section{Electronic structure of superlattices of graphene and hexagonal boron nitride}

\author{
T. P. Kaloni, Y. C. Cheng and U. Schwingenschlögl* \\ Received 30th September 2011, Accepted 19th October 2011 \\ DOI: 10.1039/c1jm14895h
}

\begin{abstract}
We study the electronic structure of superlattices consisting of graphene and hexagonal boron nitride slabs, using ab initio density functional theory. We find that the system favors a short C-B bond length at the interface between the two component materials. A sizeable band gap at the Dirac point is opened for superlattices with single graphene layers but not for superlattices with graphene bilayers. The system is promising for applications in electronic devices such as field effect transistors and metal-oxide semiconductors.
\end{abstract}

\section{Introduction}

Graphene is a two-dimensional crystalline material, consisting of a honeycomb structure with two crystallographically equivalent carbon atoms in its primitive unit cell. Two electronic bands with $\mathrm{p}_{z}$ character cross the Fermi energy precisely at the $\mathrm{K}$ point in momentum space. Consequently, undoped graphene is a zero gap semiconductor. The linear dispersion of the bands at the $\mathrm{K}$ point reflects Dirac fermions, zero mass quasiparticles. An important property of graphene is its unusual quantum Hall effect. ${ }^{1,2}$ The synthesis of single layer graphene by Novoselov et $a{ }^{3}{ }^{3}$ in addition has opened a new way to construct field effect transistors (FETs $)^{4,5}$ as well as metal-oxide field effect transistors (MOSFETs). ${ }^{6}$ Since typical sizes of graphene flakes are a few micrometres, they are easy to attach and therefore useful for device applications.

However, the lack of a finite band gap is a major obstacle on the way to an application. Hence, huge efforts have been devoted to the creation of a tunable gap in graphene systems. Different approaches have been proposed, such as the use of bilayer graphene, ${ }^{16}$ nanowires, ${ }^{17}$ substrates, ${ }^{18,19}$ twisted graphene, ${ }^{20}$ and superlattices with boron nitride. ${ }^{21,22}$ In bilayer graphene it is possible to open a band gap of around $250 \mathrm{meV}$ when the symmetry between the two layers is broken, e.g., by applying a bias potential. In graphene nanowires band gaps of 20 to $24 \mathrm{meV}$ have been found, arising from the lateral constriction of the electron in the wire. The size of the band gap depends on the shape of the wire edges. For graphene attached to various substrates band gaps of 26 to $52 \mathrm{meV}$ have been reported. In twisted graphene band gaps of 240 to $270 \mathrm{meV}$ have been observed, depending on the rotation angle. Finally, interaction between boron nitride and graphene opens a band gap of around $18 \mathrm{meV}$. In all these cases the two carbon atoms in the unit cell become inequivalent. This breaks the sublattice symmetry and generates an intrinsic robust mass of the quasiparticles.

PSE Division, KAUST, Thuwal, 23955-6900, Kingdom of Saudi Arabia. E-mail: udo.schwingenschlogl@kaust.edu.sa; Tel: +966 (0) 544700080
Hexagonal boron nitride (h-BN) is a widely known wide band gap semiconductor. Its structure is similar to graphite, ${ }^{7}$ consisting of hexagonal rings, where every boron atom is bound to three nitrogen atoms in the hexagonal basis plane, and vice versa. The strong directional bonding between adjacent intraplanar atoms comes along with charge transfer from the boron to the nitrogen atoms. Interplanar bonding is very weak with no directional bonds present, resulting in a mixture of electrostatic attraction between oppositely charged ions in adjacent planes and van der Waals bonding, similar to graphite. The band structure has been studied experimentally ${ }^{8-11}$ and theoretically. ${ }^{12-14}$ However, from the theoretical point of view the electronic properties of h-BN, especially the nature of the band gap, are not yet settled. Both direct and indirect band gaps have been reported, with values ranging between 3.6 and $7.0 \mathrm{eV} .^{15}$

In this paper we apply a multilayer scheme to study the opening of the band gap. We address the stability, cohesive energy, band structure, and (local) density of states ((L)DOS) for a series of multilayers of h-BN and graphene within the framework of density functional theory. Since the lattice parameters of $\mathrm{h}-\mathrm{BN}$ and graphene are close to each other, h-BN is a suitable choice for application in a superlattice structure with graphene. We will show that the interaction between the carbon atoms and the h-BN can open a band gap, which makes the system interesting for application in electronic devices. For instance, the room temperature $I-V$ characteristics of graphene based FETs can be improved and MOSFETs can be constructed.

\section{Method of calculation}

We have carried out first principles calculations, applying the self-consistent plane wave pseudopotential scheme implemented in the Quantum ESPRESSO code. ${ }^{23}$ For the exchange correlation functional we use the generalized gradient approximation (GGA) ${ }^{24}$ and take into account the van der Waals interaction via the B97-D functional (GGA+vdW). ${ }^{25,26}$ This functional includes damped atom-pairwise dispersion corrections of the form $C^{6} R^{-6}$, where $C$ is the dispersion coefficient and $R$ the interatomic 
distance. The B97-D functional has been designed for noncovalent complexes (including large vdW systems) by optimizing the atomic parameters for almost the entire periodic table. It has shown to yield structures and interaction energies which deviate only insignificantly from coupled-cluster results. ${ }^{25,26}$ However, since it still suffers from the GGA self-interaction error, reaction barriers are systematically underestimated. For graphite a slight overbinding has been confirmed.

For each structural configuration we have performed an optimization (force minimization) of the $c$ lattice constant and of the atomic coordinates. We have employed different k-meshes for the Brillouin zone in order to check the convergence with respect to the fineness of the k-mesh. The subsequent results are based on a Monkhorst-Pack ${ }^{27} 8 \times 8 \times 8$ k-mesh. We obtain a convergence in total energy of $10^{-9} \mathrm{Ry}$. Moreover, the cut-off energy for the plane wave expansion is obtained by a convergence test for the total energy. We find an 100 Ry kinetic energy cut-off to be sufficient. ${ }^{28}$ The equilibrium lattice constants are

Table 1 Name, stacking order, interface arrangement, band gap at the $\mathrm{K}$ point (in $\mathrm{meV}$ ), and structure sketch of the superlattice configurations under consideration

\begin{tabular}{|c|c|c|c|c|c|}
\hline Name & Stacking & Interface & $\begin{array}{l}\text { Gap } \\
\text { (GGA) }\end{array}$ & $\begin{array}{l}\text { Gap } \\
(G G A+v d W)\end{array}$ & Structure \\
\hline$(1,1)_{a}$ & $\mathrm{AB}$ & C-B, C-hollow & 70 & 111 & \\
\hline$(1,1)_{b}$ & $\mathrm{AB}$ & C-N, C-hollow & 57 & 157 & \\
\hline$(1,1)_{c}$ & AA & $\mathrm{C}-\mathrm{B}, \mathrm{C}-\mathrm{N}$ & 95 & 325 & \\
\hline$(2,1)_{a}$ & $\mathrm{ABA}$ & C-B, C-hollow & 23 & 119 & \\
\hline$(2,1)_{b}$ & AAA & $\mathrm{C}-\mathrm{B}, \mathrm{C}-\mathrm{N}$ & 41 & 227 & \\
\hline$(2,1)_{c}$ & $\mathrm{AAB}$ & C-B, C-hollow & 15 & 80 & \\
\hline$(2,1)_{d}$ & $\mathrm{ABA}$ & C-N, C-hollow & 31 & 76 & \\
\hline$(2,1)_{e}$ & $\mathrm{ABB}$ & $\mathrm{C}-\mathrm{B}, \mathrm{C}-\mathrm{N}$ & 30 & 185 & \\
\hline$(3,1)_{a}$ & $\mathrm{ABAB}$ & $\mathrm{C}-\mathrm{B}, \mathrm{C}-\mathrm{N}$ & 64 & 121 & \\
\hline$(3,1)_{b}$ & ABAA & $\mathrm{C}-\mathrm{B}, \mathrm{C}-\mathrm{N}$ & 170 & 318 & \\
\hline$(2,2)_{a}$ & $\mathrm{ABAB}$ & - & 0 & 0 & \\
\hline
\end{tabular}

obtained by minimizing the total energy and are used for calculating the band structure, cohesive energy, and (L)DOS. The experimental values of the lattice constants are $2.46 \AA^{29}$ and $6.7 \AA^{30}$ for graphene and $2.50 \AA$ and $6.66 \AA^{31}$ for h-BN. The $a$ lattice constant of our supercells is set to $2.466 \AA$, while the $c$ lattice constant is optimized. For the GGA we obtain values of $7.57 \AA, 11.42 \AA$, and $13.56 \AA$ for 2,3 , and 4 layers, respectively, while these values amount to $6.85 \AA, 9.93 \AA$, and $13.96 \AA$ for the GGA+vdW approach.

\section{Structural setup and stability}

On the basis of the above structural parameters, we construct a series of multilayers, as summarized in Table 1 . We label the different configurations $(n, m)$ according to the numbers $n$ of h$\mathrm{BN}$ layers and $m$ of graphene layers. The $(1,1),(2,1)$, and $(3,1) /$ $(2,2)$ configurations have four, six, and eight atoms in the supercell, respectively. In the following, we will not discuss thicker slabs, since there do not appear any new features. The cohesive energy per atom is calculated as $E_{\text {coh }}=\left(E_{\text {cell }}-\sum E_{\text {atom }}\right) / n$, where $E_{\text {cell }}$ is the total energy of the unit cell containing $n$ atoms and $E_{\text {atom }}$ is the total energy of the isolated atoms. $E_{\text {atom }}$ can be calculated by placing a single atom in a large supercell. The convergence with respect to the size of the supercell has been tested. The resulting values of the cohesive energy are $8.94 \mathrm{eV} /$ atom, $8.87 \mathrm{eV} /$ atom, and $8.83 \mathrm{eV} /$ atom, respectively, for the $(1,1)_{a},(2,1)_{a}$, and $(3,1)_{a}$ configurations using the GGA. Inclusion of the van der Waals interaction leads to modified values of $9.56 \mathrm{eV} /$ atom, $9.49 \mathrm{eV} / \mathrm{atom}$, and $9.46 \mathrm{eV} /$ atom but preserves the energetic order. The cohesive energy of the $(2,2)_{a}$ configuration is high, amounting to $8.94 \mathrm{eV} /$ atom (GGA) and $9.56 \mathrm{eV} / \mathrm{atom}(\mathrm{GGA}+\mathrm{vdW})$. The configurations under investigation are illustrated in Fig. 1. Note that the subscript in the notation denotes the stacking according to the leftmost column in Table 1 .

Out of the possible structures in Table 1, the lowest-energy configurations have a carbon atom next to boron and another next to the center of the h-BN ring (the hollow site). For these configurations the cohesive energies likewise are minimal. The $\mathrm{C}-$ $\mathrm{C}$ bond is strong $\sigma$-type, whereas the $\mathrm{B}-\mathrm{N}$ bond is rather ionic in nature, as a consequence of bonding between the electronegative (a)

(c)

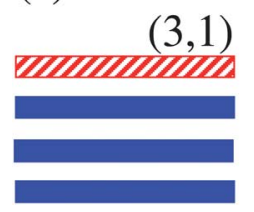

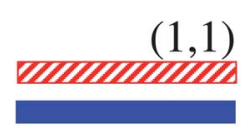

(b)

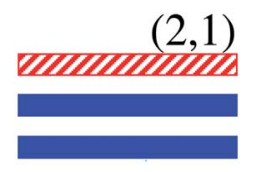

(d)

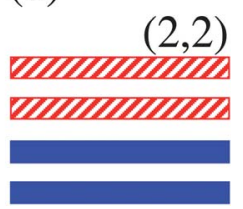

Fig. 1 Schematic illustration of the stacking in the $(1,1),(2,1),(3,1)$, and $(2,2)$ configurations. Full blue bars mark h-BN layers, patterned red bars mark graphene layers. 
nitrogen and the electropositive boron. A similar ionic bonding nature applies to both the $\mathrm{B}-\mathrm{C}$ and $\mathrm{C}-\mathrm{N}$ bonds. Due to the different electronegativities of boron (2.04), carbon (2.55), and nitrogen (3.04) there is a transfer of charge from boron to carbon and from carbon to nitrogen, where the interaction between carbon and boron dominates. A Löwdin analysis results in a charge gain of 0.36 electrons for nitrogen and charge losses of 0.41 and 0.02 electrons for boron and carbon, respectively. In summary, the most stable configuration is characterized by a short B-C distance.

\section{Electronic structure}

For the energetically favourable structures $(1,1)_{a},(2,1)_{a}$, and $(3,1)_{a}$, we compute the electronic band structure, DOS, and LDOS, see Fig. 2 to 4 . For the wide band gap insulator h-BN ( $4.7 \mathrm{eV}$ at the $\mathrm{K}$ point $^{32}$ ) our calculated GGA value of the direct band gap is $4.90 \mathrm{eV}$ and of the indirect band gap is $4.82 \mathrm{eV}$. In Fig. 2 to 4 we present the band structures of the $(1,1)_{a},(2,1)_{a}$, and $(3,1)_{a}$ configurations along the $\Gamma-\mathrm{K}-\mathrm{M}-\Gamma$ path. For the GGA we find band gaps of $70 \mathrm{meV}, 23 \mathrm{meV}$, and $64 \mathrm{meV}$, respectively, while inclusion of the van der Waals interaction alters these values to $111 \mathrm{meV}, 119 \mathrm{meV}$, and $121 \mathrm{meV}$.

The calculated band structures are compared to h-BN and pristine graphene in Fig. 2 to 4 . The DOS and LDOS are addressed on the right side of the figures. For the multilayers we obtain a vanishing DOS at the Fermi level, i.e., a finite band gap. In contrast, for pristine graphene we observe the expected perfect Dirac cone, ${ }^{33}$ whereas for h-BN we observe the large band gap mentioned above. The $\pi$ and $\pi^{*}$ bands near the Fermi level are due to the $\mathrm{sp}^{2}$ hybridized $\mathrm{C} \mathrm{p}_{z}$ orbitals, whereas the $\mathrm{p}_{x}$ and $\mathrm{p}_{y}$ contributions are small. There are almost no contributions from boron and nitrogen in the vicinity of the Fermi level.

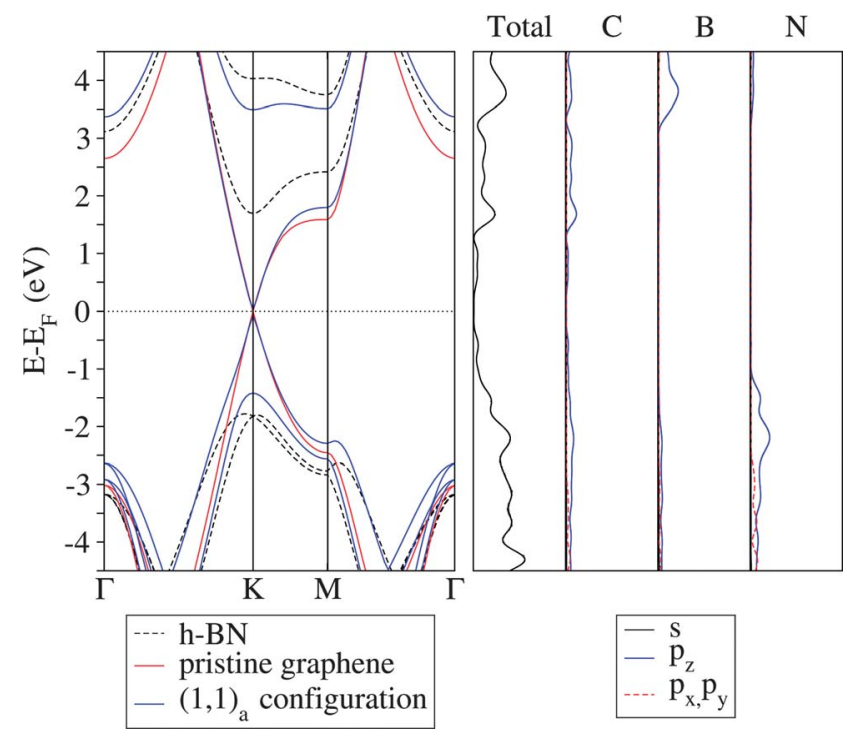

Fig. 2 Left: GGA+vdW band structure of the $(1,1)_{a}$ configuration, compared to pristine graphene and h-BN along the $\Gamma-\mathrm{K}-\mathrm{M}-\Gamma$ path. Right: Corresponding total density of states and contribution of the carbon $(\mathrm{C})$, boron $(\mathrm{B})$, and nitrogen $(\mathrm{N})$ atoms, respectively. The zero of the energy axis is set to the middle of the energy gap between the valence and conduction bands.

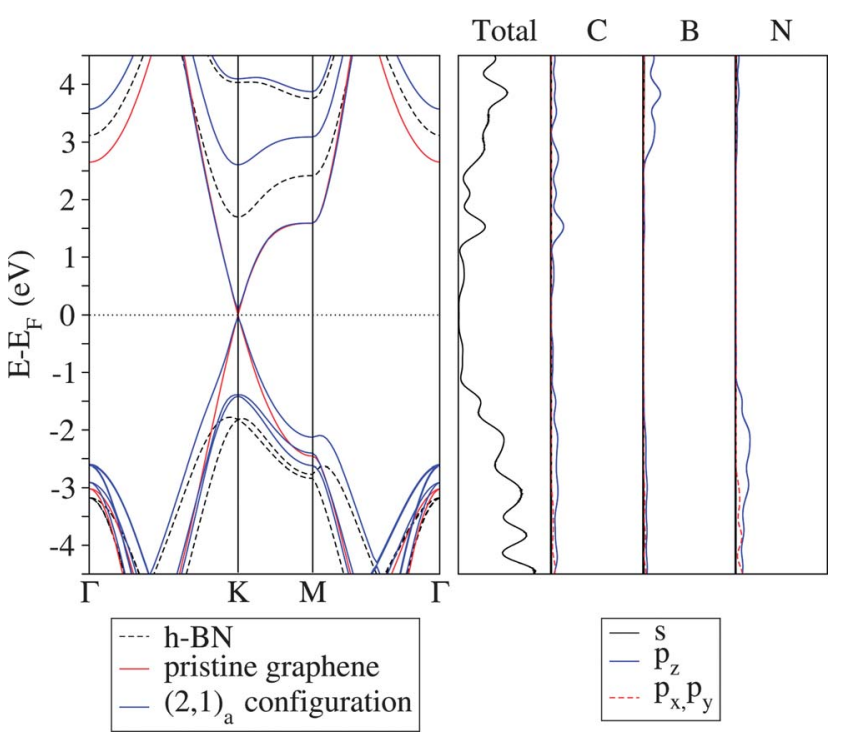

Fig. 3 Analogous to Fig. 1, but for the $(2,1)_{a}$ configuration.

The carbon $\pi$ and $\pi^{*}$ states interact with the boron and nitrogen $\pi$ and $\pi^{*}$ states (tracing back to the respective $\mathrm{p}_{z}$ orbitals), which breaks the symmetry and opens a band gap. The boron contributions dominate the $\pi^{*}$ state, while the nitrogen contributions dominate the $\pi$ state. In the case of pristine graphene, the $\pi$ and $\pi^{*}$ bands meet exactly at the Fermi level, forming a Dirac cone. These bands are equally contributed by the $\mathrm{sp}^{2}$ hybridized $\mathrm{p}_{z}$ orbitals of the two carbon atoms in the graphene unit cell. In contrast, in the h-BN case the $\pi^{*}$ band is dominated by boron and thus shifts to a higher energy, while the $\pi$ band is dominated by nitrogen and shifts to a lower energy to accommodate an additional electron. By the reduction of the symmetry, the Dirac cone is no longer preserved and quadratic bands arise. From the results in Fig. 2 to 4 it is clear that a finite band gap is established in multilayers consisting of a single graphene layer and one or more h-BN layers.

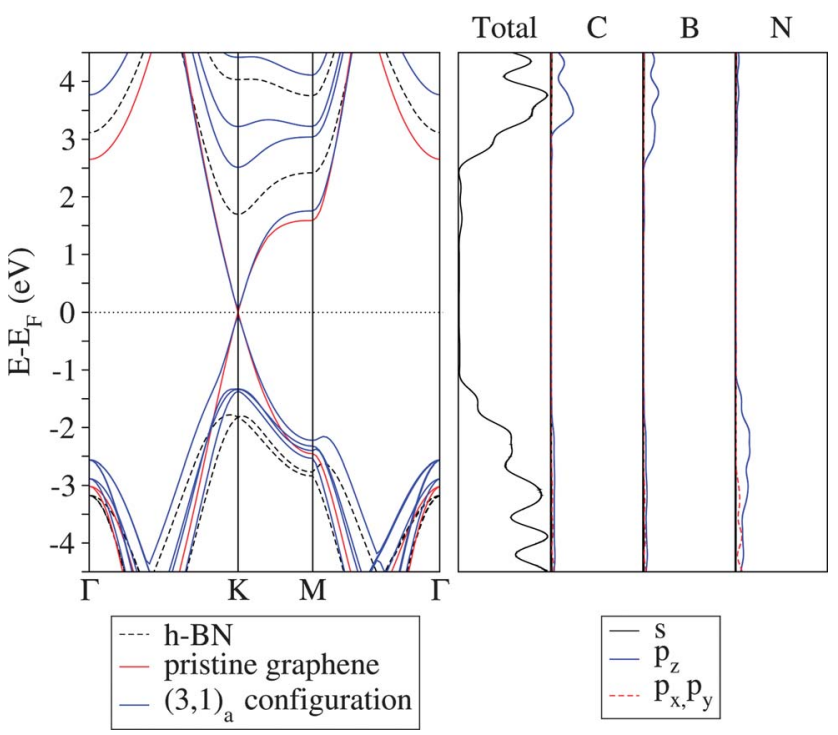

Fig. 4 Analogous to Fig. 1, but for the $(3,1)_{a}$ configuration. 


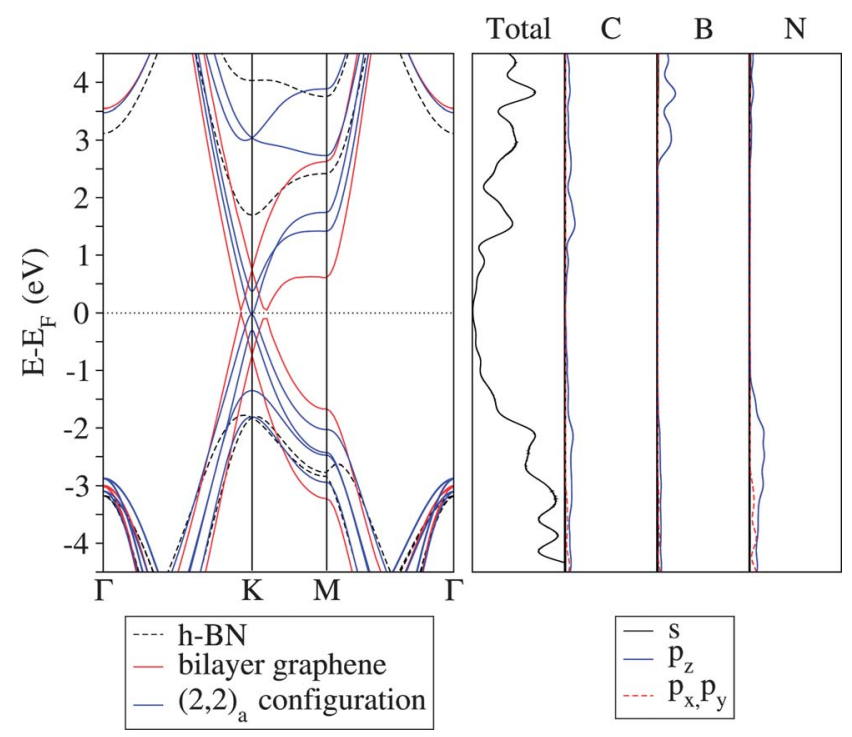

Fig. 5 Analogous to Fig. 1, but for the $(2,2)_{a}$ configuration.

In Fig. 5 we show the band structure of the $(2,2)_{a}$ configuration along the $\Gamma-\mathrm{K}-\mathrm{M}-\Gamma$ path. The calculated band structure here is compared to h-BN and bilayer graphene. Note that we have four inequivalent carbon atoms due to the interaction between the two graphene layers and the h-BN. The DOS and LDOS are addressed on the right side of Fig. 5, which demonstrates that the $\pi$ and $\pi^{*}$ bands near the Fermi level are due to the $\mathrm{sp}^{2}$ hybridised $\mathrm{C} \mathrm{p}_{z}$ orbitals with only minor $\mathrm{p}_{x}$ and $\mathrm{p}_{y}$ contributions. Again, there are almost no contributions from boron and nitrogen around the Fermi level. In contrast to bilayer graphene, the $(2,2)_{a}$ configuration shows no finite band gap as a consequence of the graphene-graphene interaction. Still, the nature of the bands at the $\mathrm{K}$ point is parabolic instead of linear. The $(2,2)_{a}$ configuration therefore remains a zero gap semiconductor. We conclude that it is not possible to open a band gap in superlattices with bilayer graphene.

\section{Conclusion}

Our first principles calculations show that among the superlattices of graphene and h-BN the favourable configurations minimize the $\mathrm{C}-\mathrm{B}$ bond length due to strong interaction between boron and carbon. For a single graphene layer alternating with a single h-BN layer the two carbon atoms of the graphene unit cell become inequivalent due to the interaction with the h-BN. Therefore, a band gap of $111 \mathrm{meV}$ opens at the $\mathrm{K}$ point. When the number of h-BN layers is increased the size of the band gap at the $\mathrm{K}$ point decreases. However, the decrease saturates already at a h-BN slab thickness of three layers. In contrast to the case of a single layer, configurations with bilayer graphene do not exhibit band gaps. In conclusion, we predict finite and tunable band gaps for superlattices in which a single graphene layer alternates with h-BN slabs of variable thickness.

\section{References}

1 K. S. Novoselov, A. K. Geim, S. V. Morozov, D. Jiang, M. I. Katsnelson, S. V. Dubonos, I. V. Grigorieva and A. A. Firsov, Nature, 2005, 438, 197.

2 Y. B. Zhang, Y. W. Tan, H. L. Stormer and P. Kim, Nature, 2005, 438, 2001.

3 K. S. Novoselov, A. K. Geim, S. V. Morozov, D. Jiang, M. I. Katsnelson, S. V. Dubonos, I. V. Grigorieva and A. A. Firsov, Science, 2004, 306, 666.

4 F. Schwierz, Nat. Nanotechnol., 2010, 5, 487.

5 X. Wang, X. Li, Y. Yoon, P. K. Weber, H. Wang, J. Guo and H. Dai, Science, 2009, 324, 768.

6 T. J. Echtermeyer, M. C. Lemme, J. Bolten, M. Baus, M. Ramsteiner and H. Kurz, Eur. Phys. J. Spec. Top., 2007, 148, 19.

7 N. Ooi, A. Raikar, L. Lindsley and J. B. Adams, J. Phys.: Condens. Matter, 2006, 18, 97.

8 J. Zupan and D. Kolar, J. Phys. Condens. Matter, 1972, 5, 3097.

9 E. Tegeler, N. Kosuch, G. Wiech and A. Faessler, Phys. Status Solidi $B, 1979,91,223$.

10 V. V. Loptan and F. V. Konusov, J. Phys. Chem. Solids, 1992, 53, 847.

11 V. L. Solozhenko, A. G. Lazarenko, J. P. Petitet and A. V. Kanaev, J. Phys. Chem. Solids, 2001, 62, 1331.

12 J. Robertson, Phys. Rev. B, 1984, 29, 2131.

13 T. K. Park, K. Terakura and N. Hamada, J. Phys. Condens. Matter, 1987, 20, 1241.

14 L. Liu, Y. P. Feng and Z. X. Shen, Phys. Rev. B: Condens. Matter, 2003, 68, 104102.

15 K. Watanabe, T. Taniguchi and H. Kanda, Nat. Mater., 2004, 3, 404.

16 C.-H. Park and S. G. Louie, Nano Lett., 2010, 10, 426.

17 Y. W. Son, M. L. Cohen and S. G. Louie, Phys. Rev. Lett., 2006, 97, 216803.

18 S. Y. Zhou, G.-H. Gweon, A. V. Fedorov, P. N. First, W. A. de Heer, D.-H. Lee, F. Guinea, A. H. Castro Neto and A. Lanzara, Nat. Mater., 2007, 6, 770.

19 P. Shemella and S. K. Nayak, Appl. Phys. Lett., 2009, 94, 032101.

20 G. Li, A. Luican, J. M. B. Lopes dos Santos, A. H. Castro Neto, A. Reina, J. Kong and E. Y. Andrei, Nat. Phys., 2010, 6, 109.

21 G. Giovannetti, P. A. Khomyakov, G. Brocks, P. J. Kelly and J. van den Brink, Phys. Rev. B: Condens. Matter Mater. Phys., 2007, 76, 073103.

22 L. Ci, L. Song, C. Jin, D. Jariwala, D. Wu, Y. Li, A. Srivastava, Z. F. Wang, K. Storr, L. Balicas, F. Liu and P. M. Ajayan, Nat. Mater., 2010, 9, 430.

23 P. Giannozzi, et al., J. Phys.: Condens. Matter, 2009, 21, 395502; Quantum ESPRESSO, Version 4.1.2.

24 J. P. Perdew, K. Burke and M. Ernzerhof, Phys. Rev. Lett., 1996, 77, 3865.

25 S. Grimme, J. Comput. Chem., 2006, 27, 1787.

26 V. Barone, M. Zerbetto and A. Polimeno, J. Comput. Chem., 2009, 30, 934.

27 H. J. Monkhorst and J. D. Pack, Phys. Rev. B: Solid State, 1976, 13, 5188.

28 Y.C. Cheng and U. Schwingenschlögl, Appl. Phys. Lett., 2010, 97, 193304; Y. C. Cheng, T. P. Kaloni, G. S. Huang and U. Schwingenschlögl, Appl. Phys. Lett., 2011, 99, 053117; T. P. Kaloni, Y. C. Cheng, R. Faccio and U. Schwingenschlögl, J. Mater. Chem., 21, 18284.

29 V. Verma, V. K. Jindal and K. Dharamvir, Nanotechnology, 2007, 18, 435711.

30 N. N. Greenwood and A. Earnshaw, Chemistry of the Elements, p. 307 (Pargamon, Oxford, 1984)

31 A. Bosak, J. Serrano and M. Krisch, Phys. Rev. B: Condens. Matter Mater. Phys., 2006, 73, 041402(R).

32 B. Arnaud, S. Lebegue, P. Rabiller and M. Alouani, Phys. Rev. Lett., 2006, 96, 026402.

33 M. A. Khan, M. A. Mukaddam and U. Schwingenschlögl, Chem. Phys. Lett., 2010, 498, 157. 